ARTICLE

\title{
Single-layered organic photovoltaics with double cascading charge transport pathways: $18 \%$ efficiencies
}

Ming Zhang ${ }^{1,7}$, Lei Zhu (1) 1,7, Guanqing Zhou' ${ }^{1,7}$, Tianyu Hao ${ }^{1}$, Chaoqun Qiu', Zhe Zhao1, Qin Hu (1) ${ }^{2}$, Bryon W. Larson (10 ${ }^{3}$, Haiming Zhu (10 4, Zaifei Ma ${ }^{5}$, Zheng Tang ${ }^{5}$, Wei Feng ${ }^{6}$, Yongming Zhang ${ }^{1,6}$, Thomas P. Russell ${ }^{2}$ \& Feng Liu (10) 1,6凶

The chemical structure of donors and acceptors limit the power conversion efficiencies achievable with active layers of binary donor-acceptor mixtures. Here, using quaternary blends, double cascading energy level alignment in bulk heterojunction organic photovoltaic active layers are realized, enabling efficient carrier splitting and transport. Numerous avenues to optimize light absorption, carrier transport, and charge-transfer state energy levels are opened by the chemical constitution of the components. Record-breaking PCEs of $18.07 \%$ are achieved where, by electronic structure and morphology optimization, simultaneous improvements of the open-circuit voltage, short-circuit current and fill factor occur. The donor and acceptor chemical structures afford control over electronic structure and chargetransfer state energy levels, enabling manipulation of hole-transfer rates, carrier transport, and non-radiative recombination losses.

\footnotetext{
${ }^{1}$ Frontiers Science Center for Transformative Molecules, In-situ Center for Physical Science, and Center of Hydrogen Science, School of Chemistry and Chemical Engineering, Shanghai Jiao Tong University, Shanghai 200240, People's Republic of China. ${ }^{2}$ Department of Polymer Science and Engineering, University of Massachusetts, Amherst, MA 01003, USA. ${ }^{3}$ Chemistry \& Nanoscience Department, National Renewable Energy Laboratory, Golden, Colorado 80401, USA. ${ }^{4}$ Department of Chemistry, Zhejiang University, Hangzhou 310027, People's Republic of China. ${ }^{5}$ Center for Advanced Low-dimension Materials, State Key Laboratory for Modification of Chemical Fibers and Polymer Materials, College of Materials Science and Engineering, Donghua University, Shanghai 201620, People's Republic of China. ${ }^{6}$ State Key Laboratory of Fluorinated Functional Membrane Materials and Dongyue Future Hydrogen Energy Materials Company, Zibo City 256401 Shandong Province, People's Republic of China. ${ }^{7}$ These authors contributed equally: Ming Zhang,

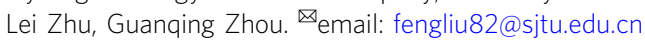


A

holistic strategy that can simultaneously improve opencircuit voltage $\left(V_{\mathrm{OC}}\right)^{1-3}$, short-circuit current $\left(J_{\mathrm{SC}}\right)^{4,5}$, and fill factor $(\mathrm{FF})^{6-9}$ has been long sought in organic photovoltaics (OPVs) to maximize power conversion efficiency (PCE). Improving all characteristics simultaneously has been hard to achieve, due to morphological and electronic structure constraints, leading to performance trade-offs. The ideal OPV device simultaneously maximizes light absorption, enhances exciton splitting, and facilitates the carrier extraction. The carrier generation kinetics must shunt energy loss channels induced by low energy chargetransfer $(\mathrm{CT})$ states to improve the $V_{\mathrm{OC}}{ }^{10,11}$. Consequently, optimizing the electronic structure of photovoltaic blends along with optimizing morphology is essential ${ }^{12-14}$. Mixtures of the midbandgap donor PM6 and the low-bandgap acceptor Y6 have shown exceptional PCE $(\sim 16 \%)$, high $J_{\mathrm{SC}}\left(\sim 25 \mathrm{~mA} \mathrm{~cm}^{-2}\right)$, and low energy loss $\left(E_{\text {loss }}, 0.5-0.6 \mathrm{eV}\right)^{15-19}$. Enhancement of the photovoltaic characteristics requires more efficient exciton splitting and carrier transport pathways in the active layer.

Here, we use quaternary blends, comprised of existing donor-acceptor pairs mixed with additional donor and acceptor components to mediate deficiencies in electronic performance or morphology, to address this challenge. It is advantageous to establish a tiered energy level alignment, to form cascading charge hopping channels that mitigate the $J_{\mathrm{SC}}$ loss by fine-tuning the charge splitting and allow manipulation of multiple chargetransfer energies to ensure a high $V_{\mathrm{OC}}$. This applies to both electron and hole transport pathways to maximize the energy gain. From the hole transport side, PM7, which has a chemical structure similar to PM6 but a deeper highest occupied molecular orbital (HOMO) energy level, can be used as an "added" donor $^{20,21}$, since it is miscible with PM6, does not disrupt the morphology, and provides a tiered cascading energy level alignment. From the electron transport side, $\mathrm{PC}_{71} \mathrm{BM}$, which is compatible with the base PM6:Y6 blends, is an acceptor with a slightly higher lowest unoccupied molecular orbital (LUMO) than Y6 that affords a cascading energy level alignment to improve electron transport and promote efficiency. Consequently, the quaternary blend strategy, where the composition can be fine-tuned to optimize device characteristics, represents a new strategy to improve PCEs.
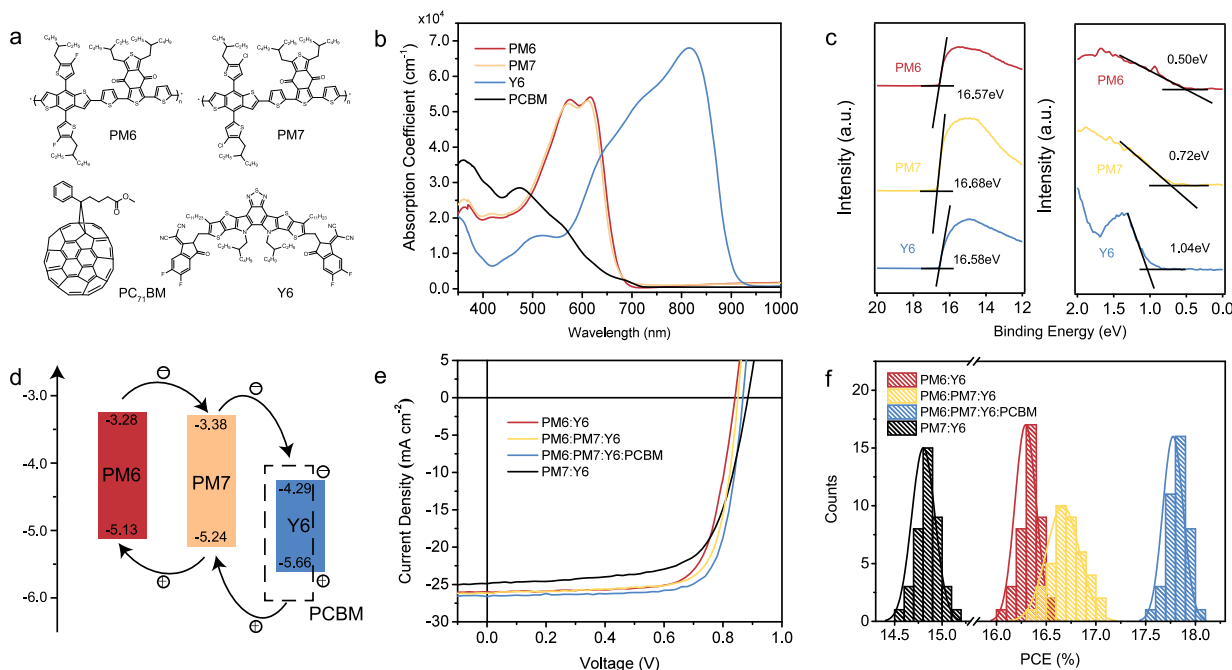

In addition to reducing the charge splitting driving force, PM7 preferentially interacts with the Y6 acceptor, effectively regulating the crystallization of Y6 to better suit carrier transport. PM7 redirects the ultrafast hole transfer from Y6 to the donor phase, forming tiered cascading energy levels that improve $J_{\mathrm{SC}}$ without further absorption gain. Adding $\mathrm{PC}_{71} \mathrm{BM}$ reduces the light absorption, yet not the current output. The higher LUMO level of $\mathrm{PC}_{71} \mathrm{BM}$ in the acceptor mixture improves the $V_{\mathrm{OC}}$ and electron transport, and offsets the absorption loss, leading to an enhancement in device performance. The synergy between the components in the PM6:PM7: Y6: $\mathrm{PC}_{71} \mathrm{BM}$ quaternary devices result in a maximum PCE of $18.07 \%$, the highest in single-layered OPV devices, with an excellent stability (81\% PCE after $1000 \mathrm{~h}$ illumination) that is $\sim 5 \%$ better than PM6:Y6 binary devices. The double cascading quaternary blend strategy is implemented in other material systems, which is reflected in similar behavior of device operation, demonstrating the novelty in the approach for OPV device fabrication. These results demonstrate the importance of manipulating the electronic structure in $\mathrm{BHJ}$ thin films, while simultaneously manipulating the morphology, opening a new route to higher efficiency OPV devices.

\section{Results}

The double cascading charge transport and device performance. Figure $1 \mathrm{a}, \mathrm{b}$ shows the chemical structure and absorption profiles of the materials used in this study. The Y6 acceptor absorbs from 700 to $950 \mathrm{~nm}$, while PM6 and PM7 donors show complementary absorption from 400 to $700 \mathrm{~nm}$. $\mathrm{PC}_{71} \mathrm{BM}$, with a much lower absorption in VIS-IR region, can mostly be considered a transport medium. The HOMO and LUMO are $-5.13 \mathrm{eV} /$ $-3.28 \mathrm{eV}$ for PM6, $-5.24 \mathrm{eV} /-3.38 \mathrm{eV}$ for PM7, $-5.66 \mathrm{eV} /-4.29$ $\mathrm{eV}$ for $\mathrm{Y} 6$, and $-6.10 \mathrm{eV} /-4.10 \mathrm{eV}$ for $\mathrm{PC}_{71} \mathrm{BM}$. The HOMO levels are accurately measured by ultraviolet photoelectron spectroscopy $(\mathrm{UPS})^{22}$, coupled with the optical band gaps to estimate the LUMO levels (Fig. 1c, d). Solar cells were prepared with a forward structure (ITO/PEDOT:PSS/active layer/PFNDI-Br/Ag). The donor to acceptor ratio was fixed at 1:1.2 (optimized conditions for PM6:Y6) to avoid light-absorption-induced performance change, and

Fig. 1 Molecular structure and photovoltaic performance of single-junction devices. a Chemical structures, b thin film absorption coefficients, and $\mathbf{c}$ UPS results of PM6 (red line), PM7 (yellow line), and Y6 (blue line). Fermi energy was determined by linear extrapolating the high binding energy portion of the spectrum, and HOMO energy level was referred to low binding energy onset. d Energy level alignment and double cascading transport pathways for quaternary system. e Current density-voltage characteristics and $\mathbf{f}$ histogram of PCE measurement for 40 devices on binary, ternary, and quaternary devices under constant incident light intensity (AM 1.5 G, $100 \mathrm{~mW} \mathrm{~cm}^{-2}$ ). $\mathbf{g}$ Normalized PCE, $V_{\mathrm{OC}}, J_{\mathrm{SC}}$, and FF (20 devices statistics) against aging time under illumination equivalent to $\sim 1$ sun for $1000 \mathrm{~h}$ (red for binary and blue for quaternary, the error bar is from the deviation of twenty samples). 
$\mathrm{PC}_{71} \mathrm{BM}$ was added separately. The concentrations of PM7 and $\mathrm{PC}_{71} \mathrm{BM}$ were varied to determine the optimal composition. Detailed performances of the devices are shown in Supplementary Fig. 1, and Supplementary Tables 1 and 2. For the PM6:PM7:Y6 ternary blends, a mixing ratio of 0.8:0.2:1.2 $\left(D_{1}: D_{2}: A_{1}\right)$ was found to be optimal, while for the PM6:PM7:Y6:PC $\mathrm{PC}_{71} \mathrm{BM}$ quaternary blends, a mixing ratio of 0.8:0.2:1.2:0.25 $\left(D_{1}: D_{2}: A_{1}: A_{2}\right)$ yielded optimum performance. The current density-voltage $(J-V)$ curves are shown in Fig. 1e, with performance detailed in Supplementary Fig. 2 and Table 1. PM6:Y6 binary devices showed a maximum PCE of $16.52 \%$, with a $V_{\mathrm{OC}}$ of $0.842 \mathrm{~V}$, a $J_{\mathrm{SC}}$ of $25.98 \mathrm{~mA} \mathrm{~cm}^{-2}$, and an FF of $75.52 \%$. Ternary devices showed a maximum PCE of $17.02 \%$, a $V_{\mathrm{OC}}$ of $0.848 \mathrm{~V}$, a $J_{\mathrm{SC}}$ of $26.17 \mathrm{~mA} \mathrm{~cm}^{-2}$, and an $\mathrm{FF}$ of $76.70 \%$. Quaternary devices showed a maximum PCE of $18.07 \%$, with a $V_{\mathrm{OC}}$ of $0.859 \mathrm{~V}$, a $J_{\mathrm{SC}}$ of $26.55 \mathrm{~mA} \mathrm{~cm}^{-2}$, and an $\mathrm{FF}$ of $79.23 \%$. The quaternary devices had a certified PCE of $17.35 \%$, subject to the calibration procedures of the National Renewable Energy Laboratory, using a $0.032 \mathrm{~cm}^{2}$ photon mask (Supplementary Fig. 3), which is the highest certified value reported for a single-layered $\mathrm{BHJ}$ device. In the ternary blends, the external quantum efficiency (EQE) improved slightly at $\sim 640 \mathrm{~nm}$ in comparison to the PM6:Y6 binary blends, as shown in Supplementary Fig. 4. In the quaternary blends, the EQE spectra of $450-600 \mathrm{~nm}$ and $650-800 \mathrm{~nm}$ improved slightly. Therefore, enhanced electron transport aided in improving the light extraction from the acceptor materials. The enhanced performance results from better charge collection channels for both electrons and holes, i.e., double cascading carrier transport pathways, which will be discussed in detail in the following section.

The stepwise-aligned energy levels lead to interesting device characteristics. The carrier recombination was determined from the dependence of the $J_{\mathrm{SC}}$ and $V_{\mathrm{OC}}$ on light intensity, as shown in Supplementary Fig. 5, Supplementary Fig. 8a, and Supplementary Table 3. A slope from $V_{\mathrm{OC}}$ vs. $P_{\text {light }}$ of $2 \mathrm{kT} / \mathrm{q}$ should be obtained if monomolecular or trap-assisted recombination dominate ${ }^{23,24}$. The recombination parameter $\alpha$, defined by $J_{\mathrm{SC}} \propto\left(P_{\text {light }}\right)^{\alpha}$, is close to unity, suggesting minimal bimolecular recombination ${ }^{25}$. From the binary to ternary to quaternary blends, $\alpha$ increased from $\sim 0.93$ to $\sim 0.94$ and to $\sim 0.96$, and the slope of $V_{\mathrm{OC}}$ vs. $P_{\text {light }}$ decreased from $1.34 \mathrm{kT} / \mathrm{q}$ to $1.18 \mathrm{kT} / \mathrm{q}$ and $1.10 \mathrm{kT} / \mathrm{q}$, respectively, consistent with the change in the FFs. These results indicate that the PM7 donor reduces trap-assisted recombination, due to a better HOMO level alignment, which will be discussed further in the following section. Adding $\mathrm{PC}_{71} \mathrm{BM}$ to the ternary blends leads to a further decrease from the electron transport side. Detailed hole and electron mobilities of the active layers with different thickness were determined using space-charge-limited current with log-log plot (Supplementary Figs. 6 and 7, and Supplementary Table 4). The space-charge-limited region was found with the slope of $(2 \pm 0.1)$, which was also fitted according to Mott-Gurney law to obtain mobility values, reflecting electron and hole transport properties ${ }^{26-28}$. It could be seen that higher mobilities correspond to better $J_{\mathrm{SC}}$ and less recombination, which seems a common phenomenon ${ }^{29,30}$. To further understand the recombination mechanism, transient photovoltage and transient photocurrent were carried out, with details shown in experimental section. Lifetimes under different $V_{\mathrm{OC}}$ conditions (tuned by changing the light intensity) could be obtained through biexponential fitting, as shown in Supplementary Fig. 9a. The lifetime of quaternary device shows a significant enhancement in the whole $V_{\mathrm{OC}}$ regime, indicating optimized device condition. The charge density as a function of $V_{\mathrm{OC}}$ for devices is calculated by differential capacitance method, with results shown in Supplementary Fig. 9b, which shows a clear exponential dependence on $V_{\mathrm{OC}}$, following $n=n_{0} e^{\gamma V_{\mathrm{OC}}}$, where $n_{0}$ is the

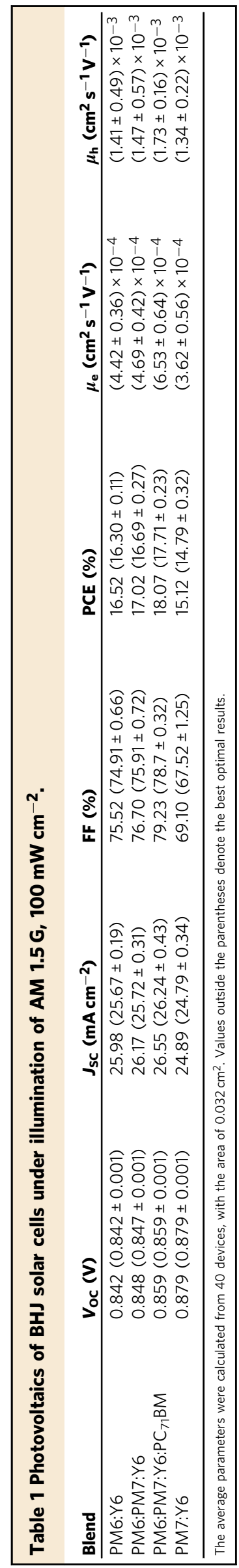


average charge density in the active layer in dark condition. The value of $\gamma$ (slope of $\ln (n)-V_{\mathrm{OC}}$ curve) for each blend was found to give similar results, in which for an ideal semiconductor, a $\gamma$ of $e / 2 k T$, equal to $19.3 \mathrm{~V}^{-1}$ at room temperature should be obtained. The deviation can be attributed to the existence of the exponential distribution of the tail states extending to the bandgap of the active layers. The improved charge density in quaternary devices echoes promoted transfer and transport properties, which offset the absorption loss. Derived charge lifetime in the devices as a function of charge density is shown in Supplementary Fig. 9c, following a power law dependence, indicating the nongeminate recombination is the dominating loss channel for carrier density under open-circuit condition. According to the above, nongeminate recombination rate coefficient can be determined, which is defined by $k(n)=\frac{1}{\tau(n) n}$, as shown in Supplementary Fig. $9 \mathrm{~d}$, and the recombination coefficient derived from Langevin theory $k_{L}=$ $\frac{q}{\varepsilon_{r} \varepsilon_{0}}\left(\mu_{n}+\mu_{p}\right)$ is calculated for comparison ${ }^{31}$. Quaternary devices show the smallest recombination rate coefficient indicating significantly suppressed nongeminate recombination. All of the devices showed a decreased recombination rate coefficient, with two orders of magnitude smaller than the Langevin recombination coefficient. The Langevin recombination rate increases with charge mobility. However, on the basis of detail balanced theory, the charge transport process in photoactive layers is much more complicated at interface, where excitons can dissociate into electrons and holes, meanwhile electrons and holes can meet to generate excitons again or annihilate directly ${ }^{32}$. The much lower recombination coefficient rate compared with Langevin theory indicates a significantly reduced nongeminate recombination loss in devices, contributing to improved devices photoelectric properties. Time-resolved microwave conductivity (TRMC) measurements were performed to characterize the free-charge generation characteristics ${ }^{33-35}$. The $\varphi \Sigma \mu$ value at the lowest absorbed flux, where we find excitation intensity-independent recombination dynamics, is used as an indicator of PV potential. As seen in Supplementary Fig. 10a, PM6:Y6 blends peak at a value of $2.3 \times 10^{-2} \mathrm{~cm}^{2} \mathrm{~s}^{-1} \mathrm{~V}^{-1}$. The addition of PM7 and $\mathrm{PC}_{71} \mathrm{BM}$, slightly increases this value to $2.4 \times 10^{-2} \mathrm{~cm}^{2} \mathrm{~s}^{-1} \mathrm{~V}^{-1}$ for the ternary and $2.9 \times 10^{-2} \mathrm{~cm}^{2} \mathrm{~s}^{-1} \mathrm{~V}^{-1}$ for the quaternary blends, indicating an increased charge generation yield. Shown in Supplementary Fig. 10b are the normalized photoconductivity transient spectra over 500 ns. PM7:Y6 shows the carrier lifetimes increase from 246 to 431 to $460 \mathrm{~ns}$ in going from the binary to ternary to quaternary blends. From a local photo-physics perspective, these improved free carrier dynamics, especially in concert with high exciton-to-charge conversion, are consistent with increasingly efficient interfacial exciton dissociation and carrier extraction, which help to improve the long-term stability of the device ${ }^{36}$.

Stability tests were performed for $1000 \mathrm{~h}$ under illumination equivalent to 1 sun, and the performance of quaternary devices maintains an $81.0 \%$ PCE, with $5.2 \% V_{\mathrm{OC}}$ loss, $5 \% J_{\mathrm{SC}}$ loss, and $10.1 \%$ FF loss (Fig. 1g and Supplementary Fig. 11), which is superior than binary devices. Temperature and humidity during the test process are shown in Supplementary Fig. 12 (average $12.5 \%$ and $41.5^{\circ} \mathrm{C}$ ). The storage stability for quaternary devices retain 97.2\% PCE after $1000 \mathrm{~h}$ aging in dark conditions (Supplementary Fig. 13), underscoring the benefits of the double cascading quaternary blends in enhancing the morphology and operation stability for long-term use. Different cathode interlayers were also investigated during stability test, which yield large variations in performances, suggesting the necessity of cathode interlayer optimization in the future.
Ultrafast hole transfer and efficient carrier transport along double cascading pathways. It is essential to understand the carrier transfer dynamics within the framework of the morphology. Femto-second transient absorption (TA) spectroscopy was used to probe the photoinduced hole transfer dynamics in the multicomponent blends ${ }^{37-39}$. The results are shown in Fig. 2, with the corresponding hole transfer times summarized in Supplementary Table 5 . The static absorption peaks for the $D$ and $A$ are spectrally well separated, so both the spectral and temporal characteristics of hole transfer dynamics can be determined. An excitation wavelength of $750 \mathrm{~nm}$ was used to selectively excite Y6. The 2D color plot of TA spectra of PM6:Y6 blend film is shown in Fig. 2a, and a few representative TA spectra at the indicated delay times are shown in Fig. 2b. With the decay of the Y6 bleach peak at $770-860 \mathrm{~nm}$, a few clear bleach peaks at $560-600 \mathrm{~nm}$ emerge in the TA spectra, matching well with the absorption features of PM6. The bleach decay process of the photoexcited Y6 agrees with the increase of the PM6 ground state bleach, confirming the ultrafast hole transfer from Y6 to PM6, as shown in Fig. 2c. We can extract the rising kinetic of PM6 bleach to represent the hole transfer process. Herein, the rising kinetic of PM6 bleach is not influenced by Y6 bleach due to well separating of static absorption peaks between PM6 and Y6, providing a clear hole transfer process. We fit the donor kinetics with a biexponential function. The hole transfer process in the four blends (PM6:Y6, PM6:PM7:Y6, PM6:PM7:Y6:PC ${ }_{71} \mathrm{BM}$, and PM7:Y6) show a fast component with $\tau_{1}$ of $\sim 0.25, \sim 0.39, \sim 0.37$, and $\sim 0.47 \mathrm{ps}$, and a slow component $\tau_{2}$ of $8.18,10.28,13.22$, and $16.36 \mathrm{ps,}$ respectively (Fig. 2d, e), and their relative contributions are shown in Supplementary Table 5. The former fast component $\tau_{1}$ can be assigned to the ultrafast exciton dissociation of $\mathrm{Y} 6$ at the donor-acceptor interface and the latter to the diffusion of excitons in Y6 toward interface before dissociation ${ }^{40-42}$. The interfacial exciton separation is more than one order of magnitude faster in comparison to the exciton diffusion. The trend in the hole transfer lifetime and the hole transfer efficiency is consistent with the values of HOMO offsets or driving force (Supplementary Fig. 14), which points out the direction of device operating principle. The presence of PM7 in the BHJ blends reduces the driving force, aligning the cascading energy levels to ensure better transport and an increase in the $J_{\mathrm{SC}}$, a trade-off with the hole transfer rate. $\mathrm{PC}_{71} \mathrm{BM}$ does not perturb the hole transfer process, suggesting close interactions between Y6 and PM6/PM7. Such results also agree well with the observation that no $\mathrm{PM} 6: \mathrm{PC}_{71} \mathrm{BM} \mathrm{CT}$ emission is seen in quaternary blends. While most studies focus on increasing absorption with the addition of more components, our findings indicate that a detailed balance between the driving force and hole transfer rate is equally important to refine the carrier generation and extraction to generate high $J_{\mathrm{SC}}$ in OSCs. We compared the polaron decay dynamics of the quaternary blend films at different pump fluences to probe the charge recombination mechanism (Supplementary Fig. 15). The difference in fluence dependence of recombination dynamics suggests that carriers recombine via the nongeminate recombination with small amount of geminate recombination (1\%), indicating of small amount of CT states formation. Timeresolved photoluminescence (TRPL) measurements were further performed. To avoid the influence of Y6 fluorescence quenching, we only probed $550-650 \mathrm{~nm}$ and pump at $515 \mathrm{~nm}$ in TRPL measurement. As illustrated in Supplementary Fig. 16 and Supplementary Table 6, PM6:PM7 blended film presents much smaller fluorescence lifetime $(\tau=672.4 \pm 9.3 \mathrm{ps})$ compared with PM6 $(1483.6 \pm 11.7 \mathrm{ps})$ and PM7 (1521.7 $\pm 13.1 \mathrm{ps})$ neat films, indicating electron transfer channel between PM6 and PM7. After blending Y6, the fluorescence lifetime is significantly decreased with a time constant of $73.9 \pm 1.23 \mathrm{ps}$, indicating a highly efficient electron 

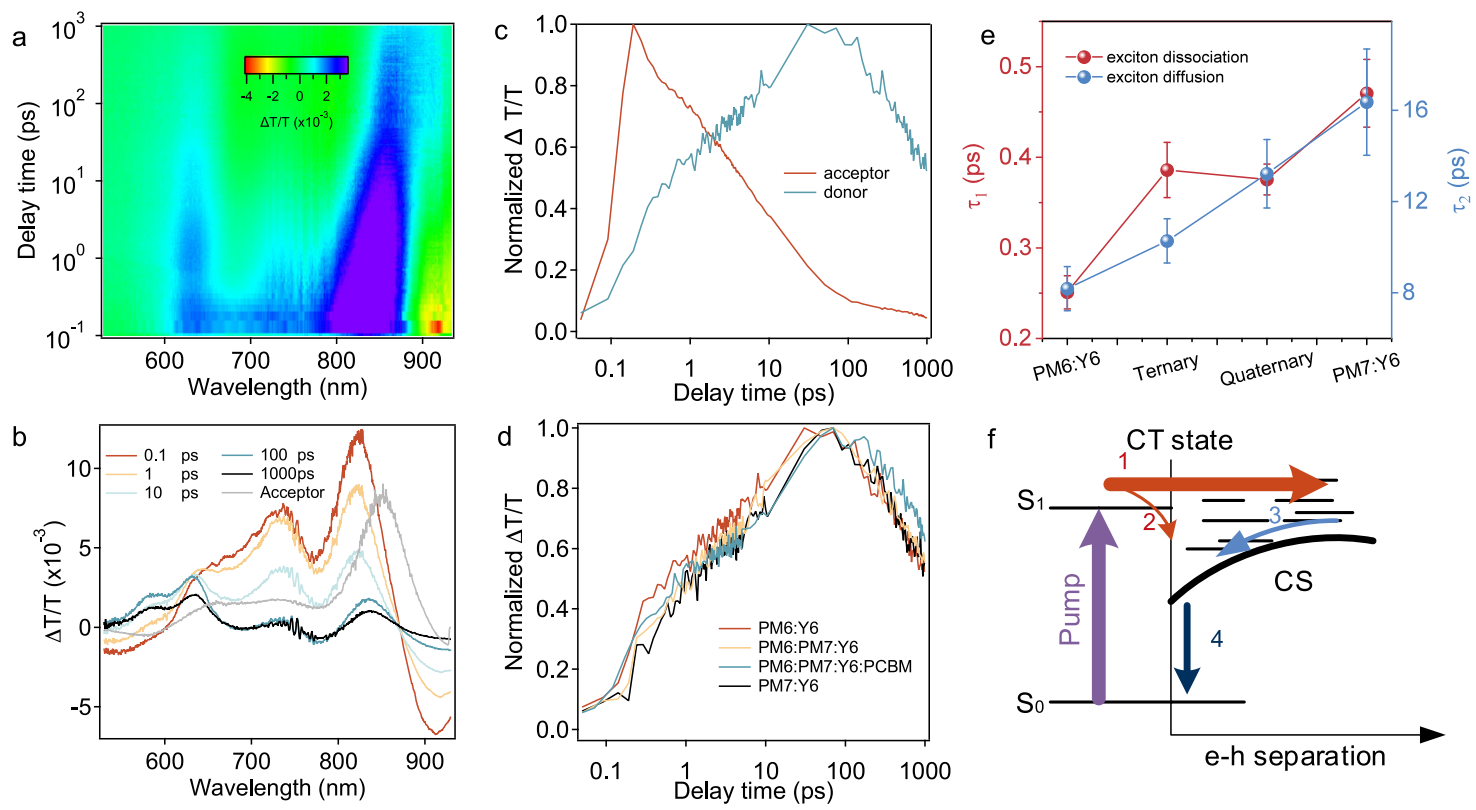

Fig. 2 Ultrafast hole transfer and efficient carrier transport along double cascading pathways. a Color plot of fs transient absorption spectra of blended film at indicated delay times under $750 \mathrm{~nm}$ excitation with a fluence $<10 \mu \mathrm{J} \mathrm{cm}{ }^{-2}$. b Representative fs TA spectra of blended films at indicated delay times. c TA kinetics in blended films showing the hole transfer process. d The hole transfer process in PM6:Y6 (red line), PM6:PM7:Y6 (yellow line), PM6:PM7: Y6:PC ${ }_{71} \mathrm{BM}$ (blue line), and PM7:Y6 (black line) blended films. e Charge-transfer time achieved through multi-exponential fitting for different blended films (the error bar is from the standard error of curve fitting). $\mathbf{f}$ Schematic of electronic states in an organic solar cell, and the excited state $\left(\mathrm{S}_{1}\right)$ can be directly separated (step 1) or form charge-transfer states (step 2), accompanied with recombination processes (steps 3 and 4).

transfer in the PM6:PM7:Y6 heterojunction. From the above results, a picture of the mechanism emerges, as shown in Fig. $2 \mathrm{f}$. The photon excitation first drives ultrafast and large amounts of free carriers accompanied with nongeminate recombination, biasing out the weak interfacially bound CT states (which are quite close in energies), along with a density-dependent recombination process (step 4).

Energy loss optimization induced by CT energy management. In solar cell devices, the $V_{\mathrm{OC}}$ decreases in going from quaternary to ternary to binary blends. This results from the energy level management, driving force, and energy loss ( $\left.E_{\text {loss }}\right)$. Highly sensitive EQE (s-EQE), electroluminescence (EL), and electroluminescence quantum efficiency (EQE-EL) measurements were performed to investigate energy loss channels ${ }^{43-45}$. Two energy loss sectors, charge generation $\left(\Delta E_{2}=E_{\mathrm{g}}-E_{\mathrm{CT}}\right)$ and charge recombination losses $\left(E_{\mathrm{CT}}-q V_{\mathrm{OC}}\right)$ were considered. The charge recombination loss could be further traced to radiative $\left(\Delta E_{1}\right)$ and non-radiative $\left(\Delta E_{3}\right)$ recombinations ${ }^{46}$. The $E_{\mathrm{CT}}$ could be obtained by fitting the sub-gap absorption of the corresponding s-EQE curve (Fig. 3b and Supplementary Fig. 17) following the Marcus theory ${ }^{43,47}$. The CT energy of the binary PM6:Y6 blend was $1.34 \mathrm{eV}$, yielding a $\Delta E_{2}=0.066 \mathrm{eV}$. PM7:Y6 blend showed a CT energy of $1.37 \mathrm{eV}$, with $\Delta E_{2}=0.061 \mathrm{eV}$. Ternary and quaternary blends showed the CT energies of 1.35 and $1.36 \mathrm{eV}$, respectively. The lowest $\Delta E_{2}$ of $0.048 \mathrm{eV}$ was obtained for quaternary films, resulting in a higher $V_{\mathrm{OC}}$. The deeper HOMO of PM7 leads to a reduced hole transfer driving force but smaller energy loss, providing one avenue for device optimization, with an optimized ternary blend composition of $20 \%$ PM7. The PM6:PC ${ }_{71} \mathrm{BM}$ mixture has a CT energy of $1.49 \mathrm{eV}$, higher than the bandgap of Y6, and thus would not introduce a low level CT state to trap electrons. Consequently, adding $\mathrm{PC}_{71} \mathrm{BM}$ to the ternary blends redefines the acceptor LUMO at a higher level, which further improves $V_{\text {OC }}$.
Figure 3a shows the normalized EL spectra of devices based on the pure materials and the BHJ films. PM6:Y6 blends show a single EL emission peak at $924 \mathrm{~nm}$, similar to that of a Y6 film $(920 \mathrm{~nm})$. When the applied current was increased from 1 to 5 mA, no EL change was observed (Supplementary Fig. 19). Thus, the CT states, if present, have a very low density. PM6: $\mathrm{PC}_{71} \mathrm{BM}$ blends have a CT emission at $980 \mathrm{~nm}$, well below the emission in the binary mixtures. However, in the quaternary blends, the EL is dominated by Y6, eliminating the PM6: PC ${ }_{71} \mathrm{BM}$ CT states. Therefore, the $\mathrm{BHJ}$ blends can be viewed as simple OLED devices, with PM6/PM7 and $\mathrm{PC}_{71} \mathrm{BM}$ acting as hole and electron transporting layers, and emission happens at the $\mathrm{Y} 6$ acceptor or PM6:Y6 interfaces. Y6, then, functions as both a photovoltaic and EL material. The energy losses due to radiative recombination $\left(\Delta E_{1}\right)$ of charge carriers can be calculated using the fit parameters from the s-EQE spectra (Fig. 3b and Supplementary Fig. 17), and the losses due to the non-radiative recombination $\left(\Delta E_{3}\right)$ were quantified by measuring the EQE-EL ${ }^{48}$. As shown in Fig. $3 c$, the emission efficiency of PM7:Y6 blend was $1.33 \times 10^{-2} \%$, much higher than that of PM6:Y6 blend $\left(5.05 \times 10^{-3} \%\right)$. Thus, for ternary blends, the additional 20\% PM7 actually reduced the energy loss caused by non-radiative recombination. The quaternary blends show a higher emission efficiency of $9.37 \times 10^{-3} \%$ in comparison to the ternary film, representing a decrease in $\Delta E_{3}$ to $0.240 \mathrm{eV}$. Non-radiative recombination can be calculated from $\Delta E_{3}=-k T \cdot \ln \left(\mathrm{EQE}_{\mathrm{EL}}\right)$. Consequently, it is important to maximize $\mathrm{EQE}_{\mathrm{EL}}$ to minimize $\Delta E_{3}$, and in the current case, the double cascading energy level alignment plays an important role. Different contributions to energy loss are shown in Fig. 3d and summarized in Supplementary Table 7. The quaternary blends showed the lowest total energy loss of $0.548 \mathrm{eV}$, in comparison to the other blends ( $0.567 \mathrm{eV}$ for PM6:Y6, $0.553 \mathrm{eV}$ for PM7:Y6, and $0.568 \mathrm{eV}$ for ternary blends). We attribute the elevated $V_{\mathrm{OC}}$ of the quaternary devices to the smallest $E_{\mathrm{g}}-E_{\mathrm{CT}}$ energy offset and the suppressed non-radiative recombination losses, due to the addition of $\mathrm{PM} 7$ and $\mathrm{PC}_{71} \mathrm{BM}$. 

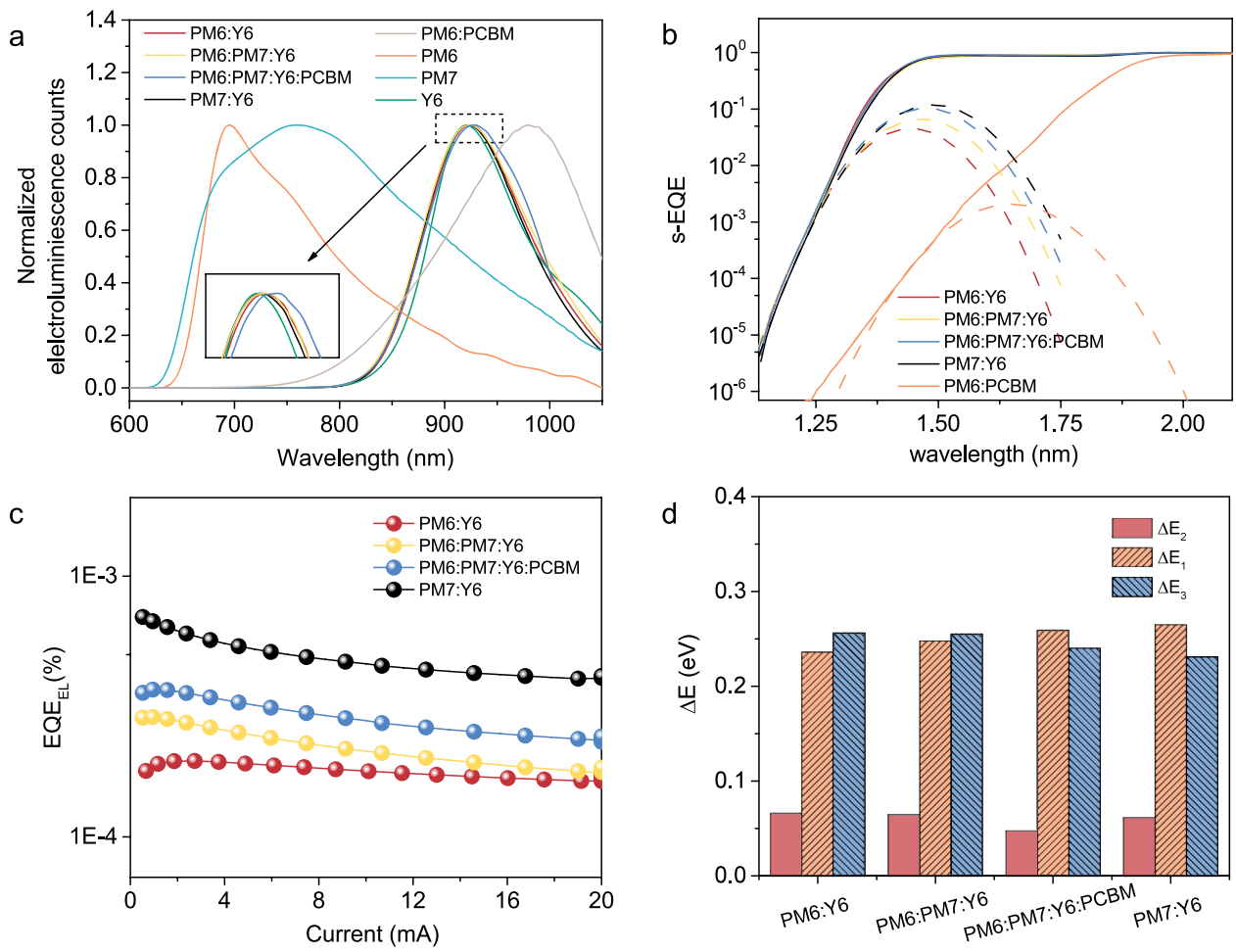

Fig. 3 Energy loss analysis. a Electroluminescence spectra of devices based on the pristine and blended films. $\mathbf{b} \mathrm{s}-\mathrm{EQE}$ and $\mathbf{c} E Q E-E L$ of the blended devices. d Energy loss histogram, including $\Delta E_{1}, \Delta E_{2}$, and $\Delta E_{3}$ of blended devices.

Thin film morphology of double cascading blends. The structure of the neat and $\mathrm{BHJ}$ thin films were determined using grazing incidence wide-angle X-ray diffraction (GIWAXS), the results of which are shown in Fig. 4 and Supplementary Fig. 20. The PM6 donor assumed a dominant face-on orientation, with a broad (100) reflection in the in-plane (IP) direction at $0.28 \AA^{-1}$ and a $\pi-$ $\pi$ stacking peak in the out-of-plane (OOP) direction at $1.69 \AA^{-1}$. The crystal coherence lengths (CCLs) for (100) and (010) were 5.27 and $1.56 \mathrm{~nm}$, respectively, as determined using the Scherrer analysis ${ }^{49,50}$. PM7 had a similar diffraction profile, with a (100) reflection in the IP direction at $0.29 \AA^{-1}$ and $(010)$ reflection in the OOP direction at $1.66 \AA^{-1}$ with CCLs of 4.52 and $1.31 \mathrm{~nm}$, respectively. Y6 films showed a $\pi-\pi$ stacking peak at $1.75 \AA^{-1}$ in the OOP direction and a lamellar stacking peaks at $0.27 \AA^{-1}$ in the IP direction. However, the banana-shaped Y6 molecules pack in a unique manner so that they can overlap by end group $\pi-\pi$ stacking to form a polymer-like conjugated backbone, and the lamellae packing is assigned to the (110) lattice plane, which is shown in Fig. 4d and Supplementary Fig. 21. Thus, Y6 assumes a tilted molecular orientation where the polymer-like backbone is tilted normal to surface, which is more efficient for charge transport.

A summary of the 2D and linecut GIWAXS profiles are shown in Fig. 4a, b, respectively. For the PM6:Y6 blends, Y6 showed well-defined IP (020) and (11-1) lattice reflections at 0.21 and $0.42 \AA^{-1}$. PM7:Y6 blends show weak crystalline order, with the Y6 (11-1) and (020) diffraction peaks absent. Even though PM6 and PM7 have similar chemical structures, PM7 interacts differently with Y6 and retards Y6 crystallization, such that only the Y6 (110) stacking can be seen. In ternary blends, the intensities of the reflections at 0.23 and $0.42 \AA^{-1}$ decreased significantly. In the quaternary blends, the Y6 reflections at 0.23 and $0.42 \AA^{-1}$ were quite weak, indicating that $\mathrm{PC}_{71} \mathrm{BM}$ also disrupts the packing of Y6. The polymer lamellar and Y6 (110) reflections could not be separated, and they were used in sum to estimate the lamellar ordering of the BHJ thin film. The polymer and Y $6 \pi-\pi$ reflections are summarized in Supplementary Table 8. Figure $4 \mathrm{c}$ shows parameters derived from the lamellar and $\pi-\pi$ stacking peaks of the different $\mathrm{BHJ}$ thin films. The quaternary blend showed the largest CCL and peak area for both the lamellar and $\pi-\pi$ stacking peaks, indicating that the overall crystallinity and crystal quality are improved for the quaternary blends, which improves carrier transport pathways. The Y6 (020) peak showed a decrease in the peak area, but an increase in the CCL in going from the binary to ternary to quaternary blends, as shown in Supplementary Fig. 22 and Supplementary Table 9. Consequently, the crystallization behavior of Y6 changed in the blends. The loss of primary axis coherence and intensity in Y6, but improvement in the (110) and $\pi-\pi$ stacking reflect an extended polymer-like conjugated backbone by adopting a twisted or screw-like packing in the multicomponent blends, providing a pathway for electron transport. A schematic of the molecular packing in the blend films is illustrated in Fig. 4e. The intimate mixing of PM6 and PM7 (as indicated by the linear dependence of the $V_{\mathrm{OC}}$ on concentration) results in the formation of a homogeneous polymer-rich phase embedded in a fibrillar network. The difference in the interactions of PM6 and PM7 with $\mathrm{Y} 6$ optimizing the crystallization of $\mathrm{Y} 6 . \mathrm{PC}_{71} \mathrm{BM}$ addition does not perturb the morphological framework of PM6:Y6 and is distributed uniformly, as evidenced by the absence of any feature characteristic of $\mathrm{PC}_{71} \mathrm{BM}$ aggregation. Thus, the plasticizing nature of $\mathrm{PC}_{71} \mathrm{BM}$ aids in the overall ordering, improving both electron and hole mobility, and a higher FF.

$\mathrm{BHJ}$ thin film phase separation was visualized using transmission electron microscopy (TEM). As shown in Supplementary Fig. 23, all the BHJ thin films showed evidence of phase separation on the tens of nanometers length scale. Resonant soft X-ray scattering (Supplementary Fig. 24) for PM6:Y6 and PM6: PM7:Y6 blends yielded an interference at a length scale of $\sim 60 \mathrm{~nm}$. The quaternary blends with different $\mathrm{PC}_{71} \mathrm{BM}$ loadings 
a
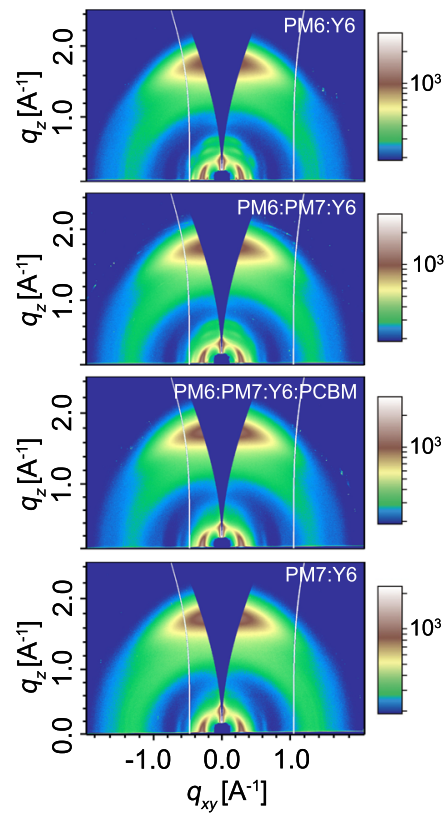

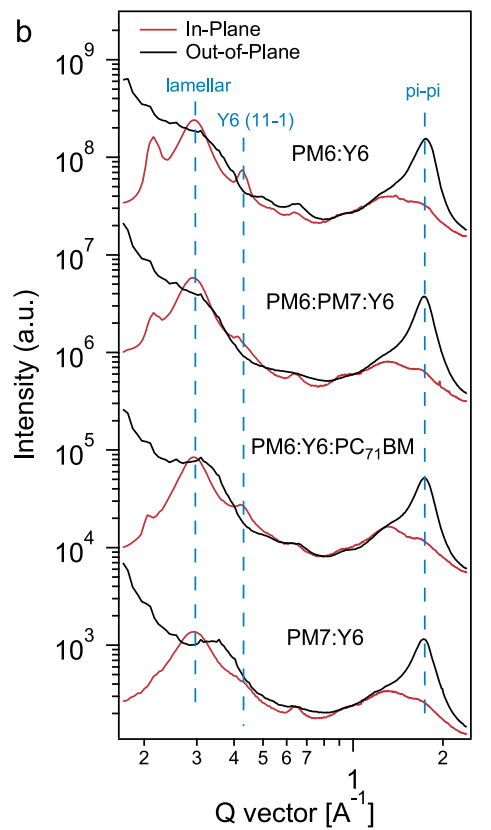

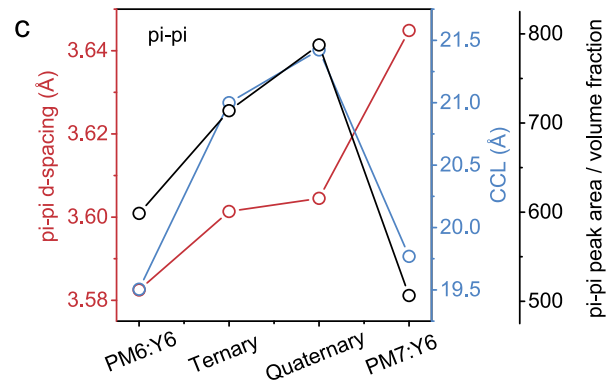

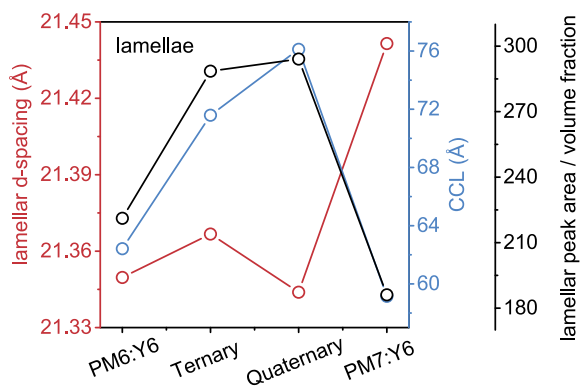

d
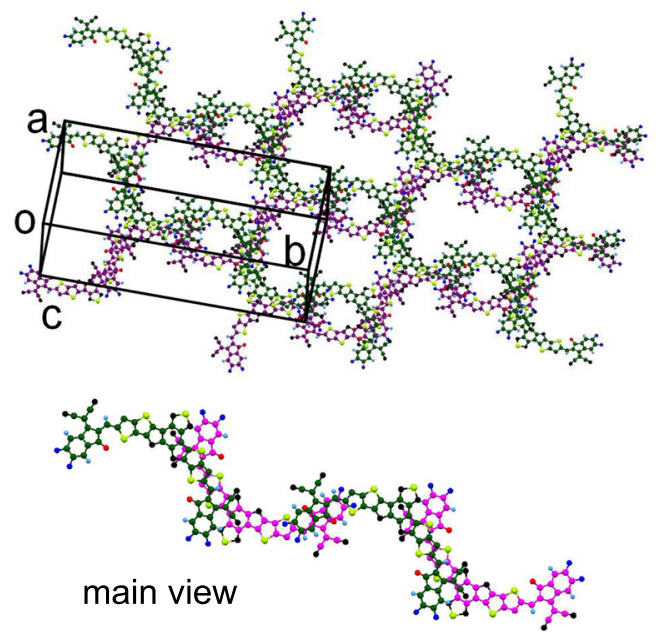

e

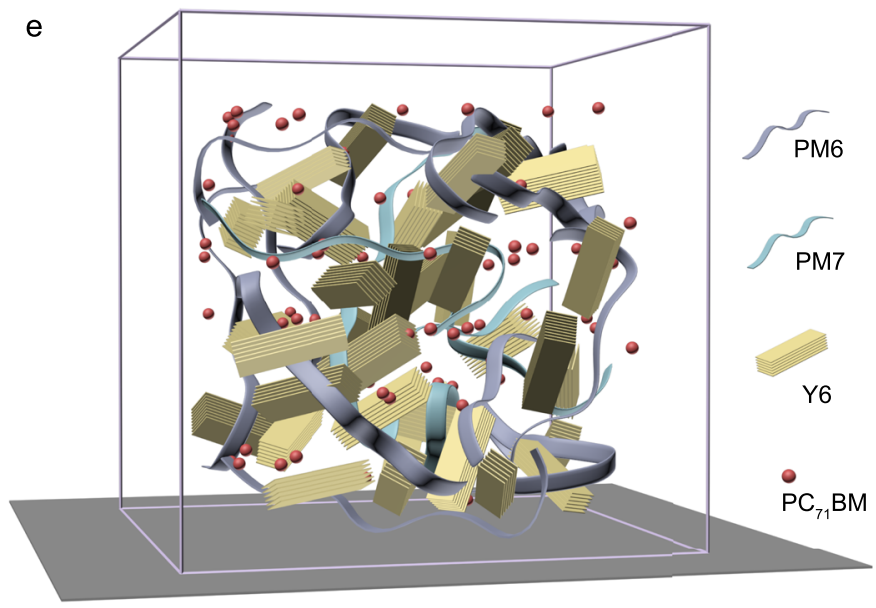

Fig. 4 Morphology optimization of thin films. a 2D GIXD patterns of the binary, ternary and quaternary blends. b Out-of-plane (black) and in-plane (red) linecut profiles of the 2D GIXD data. c D-spacing (red symbol), CCL (blue symbol) and peak area/volume fraction (black symbol) of pi-pi and lamellae diffraction peak for blended films with different composition. d Molecular packing sketch map of $\mathrm{Y} 6$ crystal. e The arrangement sketch of molecules in quaternary blended films.

(Supplementary Fig. 24b) show a shoulder gradually developing into a well-defined interference at higher $\mathrm{PC}_{71} \mathrm{BM}$ loadings, suggesting that uniform distribution of $\mathrm{PC}_{71} \mathrm{BM}$ in the mixed region enhances the scattering contrast. The uniform distribution of $\mathrm{PC}_{71} \mathrm{BM}$ in the mixture indicates that the close interactions with the other amorphous components produces a unique electronic structure with improved electron transport channels, where excited electrons in the donors can transfer onto the "LUMO" of mixed domain that are rapidly extracted. Though the HOMO of $\mathrm{PC}_{71} \mathrm{BM}$ is much deeper than that of $\mathrm{Y} 6$, a homogeneous mixing provides good contacts with the donor materials making exciton harvesting by $\mathrm{PC}_{71} \mathrm{BM}$ and $\mathrm{Y} 6$ efficient.

\section{Discussion}

The results show the importance of achieving a detailed balance between morphology, energy loss reduction, and ultrafast charge transfer kinetics in double cascading quaternary blends. The double donor strategy yields a terraced HOMO energy level alignment that facilitates hole transport in the $\mathrm{BHJ}$ blends.
Adding $\mathrm{PC}_{71} \mathrm{BM}$ that is uniformly mixed with $\mathrm{Y} 6$ produces a terraced LUMO energy level alignment, and in the HOMO levels, the longer exciton diffusion times, in comparison to the splitting times, lead to a high probability that a suitable donor/acceptor interface can be found to split excitons. The close contact between Y6 and PM7 provides an important channel where Y6 trapped exciton or hole carriers can be extracted. Such a unique double cascading energy level simultaneously improves the $V_{\mathrm{OC}}, J_{\mathrm{SC}}$, and FF. A more thorough correlation between the structure, photon-electron processes, and device performances needs to be quantified to establish a solid structure-property relationship. Shown in Fig. 5a, b are the correlations between the structural and device characteristics, Fig. $5 \mathrm{c}-\mathrm{e}$ shows relationships between charge transfer, transport, and performance, and Fig. $5 \mathrm{f}$, the correlation between energy loss and $V_{\mathrm{OC}}$. A quantitative analysis was performed using the Pearson correlation method ${ }^{51,52}$, as shown in Fig. $5 \mathrm{~g}, \mathrm{~h}$ in $3 \mathrm{D}$ and $2 \mathrm{D}$. A correlation factor of 1 , indicates a direct correlation, with decreasing values reflecting a loss in correlation. The Pearson correlation coefficient matrix is 
a
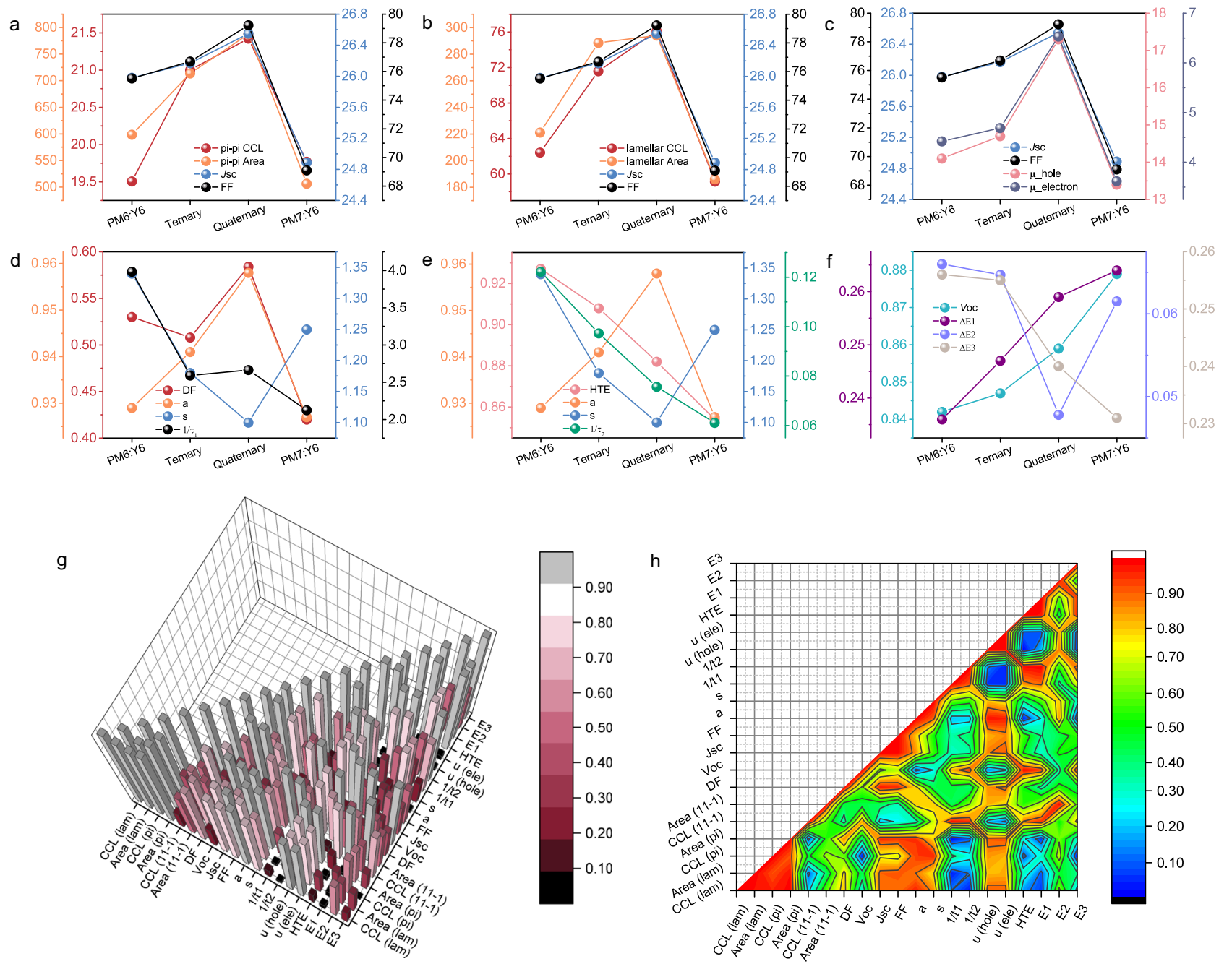

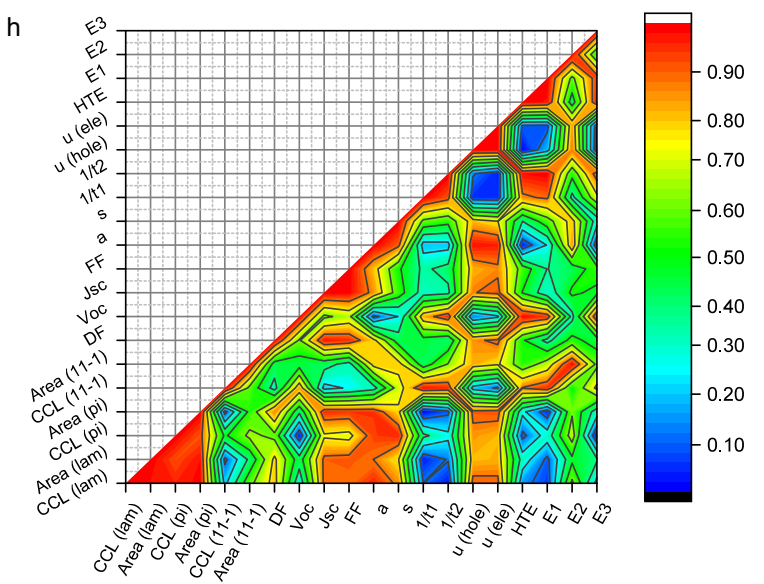

Fig. 5 Correlation of structure, photon-to-electron process, and device performance. a-f Multidimensional correlation analysis of structure-property relationships (the parameter units are following previous data). $\mathbf{g}$, h Person correlation analysis of major device and structure parameters (color bar and height both represent person correlation coefficients, CCL is the abbreviation of crystal coherence length, DF is the abbreviation of driving force, HTE is the abbreviation of hole transfer efficiency, E1-E3 represent energy loss).

shown in Supplementary Table 10. It is seen that crystallization is the major factor that accounts for $J_{\mathrm{SC}}$ and $\mathrm{FF}$ in photovoltaic devices, thus the quaternary blends reach the maximum value. Mobilities $\left(\mu_{\mathrm{h}}\right.$ and $\left.\mu_{\mathrm{e}}\right)$ are highly dependent on thin film crystallinity. $1 / \tau_{1}$ shows a strong correlation with the driving force, indicating its strong influence on exciton separation, while the trend in driving force matches well with recombination parameters, suggesting an important mechanism correlating efficient charge transfer and extraction. $V_{\mathrm{OC}}$ should show an inverted correlation with energy loss, and $\Delta E_{3}$, originating with nonradiative recombination, shows the most significant influence. Therefore, a detailed balance between morphology optimization, charge transfer efficiency, and energy loss channels controls the device output. These observations suggest a new strategy using double cascading quaternary blends for device optimization. The material properties and device performance parameters are highly interconnected, and optimizing only one parameter is not sufficient to optimize performance. Binary mixtures are effective for material screening, and ternary mixtures bring complementary absorption and energy level management into the device design, but quaternary mixtures enable a fine-tuning of both the morphology and electronic structure simultaneously. Fine-tuning such quaternary mixtures introduces a new strategy to achieve higher efficiency OPVs. We extended the double cascading quaternary mixture strategy to other well-studied NFA OPV systems (Supplementary Fig. 25 and Supplementary Table 11), with similar results been obtained as seen in PM6:PM7:Y6:PC ${ }_{71} \mathrm{BM}$ mixture, underscoring the generality of this approach.

Here, we designed multicomponents blends of polymer donors PM6 and PM7, a non-fullerene small-molecule acceptor Y6, and a fullerene acceptor $\mathrm{PC}_{71} \mathrm{BM}$. An average PCE of $16.69 \%$ was obtained when 80\% PM6 and 20\% PM7 were blended with Y6. The addition of the fourth component $\mathrm{PC}_{71} \mathrm{BM}$ significantly increased the average efficiency output to $17.71 \%\left(\mathrm{PCE}_{\max }\right.$ of $18.07 \%$ ), with a slightly improved $J_{\mathrm{SC}}$ of $26.55 \mathrm{~mA} \mathrm{~cm}^{-2}$, which could be ascribed to a fine balance between light absorption and charge extraction. The elevated $V_{\mathrm{OC}}(0.859 \mathrm{~V})$ of the quaternary device was attributed to the smallest $E_{\mathrm{g}}-E_{\mathrm{CT}}$ energy offset and the suppressed non-radiative recombination losses after the addition of PM7 and, especially, $\mathrm{PC}_{71} \mathrm{BM}$. An FF of over 0.79 was obtained, since an optimized morphology formed with balanced crystallization features that ensured better charge transport, as shown in Supplementary Fig. 26 that summarizes the holistic strategy in OPV efficiency optimization. 


\section{Methods}

Device fabrication. Organic solar cell devices with ITO/PEDOT:PSS/active layer/ $\mathrm{PFNDI}-\mathrm{Br} / \mathrm{Ag}$ regular structures were fabricated according to the following procedure. Patterned ITO glass substrates were sequential cleaned by ultrasonicating in acetone, detergent, deionized water, and isopropyl alcohol for $15 \mathrm{~min}$ each and then dried under $80^{\circ} \mathrm{C}$. The precleaned substrates were treated in an ultraviolet-ozone chamber for 15 min, then a $\sim 40 \mathrm{~nm}$ thick PEDOT:PSS (Clevious P VP AI 4083 H. C. Stark, Germany) thin film was deposited onto the ITO surface by spin-coating and baked at $150{ }^{\circ} \mathrm{C}$ for $20 \mathrm{~min}$. The blend solution with different mixing ratio $\left(14 \mathrm{mg} \mathrm{mL}^{-1}\right.$ in total) in CF (with $0.5 \% \mathrm{CN}$ solvent additive) was stirred at $25^{\circ} \mathrm{C}$ for $120 \mathrm{~min}$ in advance, and then spin-coated on top of the PEDOT:PSS layer. The prepared films were treated with thermal annealing at $85^{\circ} \mathrm{C}$ for $6 \mathrm{~min}$. After cooling to room temperature, a $\sim 5 \mathrm{~nm}$ thick of PFNDI-Br $\left(0.5 \mathrm{mg} \mathrm{mL}^{-1}\right)$ was spin-coated on the top of active layer. Then, those samples were brought into to an evaporate chamber and a $140 \mathrm{~nm}$ thick silver layer was thermally evaporated on the PFNDI-Br layer at a base pressure of $1 \times 10^{-6}$ mbar. The evaporation thickness was controlled by SQC-310C deposition controller (INFICON, Germany). Ten devices were fabricated on one substrate and the active area of each device was $0.032 \mathrm{~cm}^{2}$ defined by a shadow mask.

Reporting summary. Further information on research design is available in the Nature Research Reporting Summary linked to this article.

\section{Data availability}

The data that support the plots within this paper and other finding of this study are available from the corresponding authors upon reasonable request.

Received: 16 July 2020; Accepted: 2 December 2020;

Published online: 12 January 2021

\section{References}

1. Chen, Y. et al. Tuning Voc for high performance organic ternary solar cells with non-fullerene acceptor alloys. J. Mater. Chem. A 5, 19697-19702 (2017).

2. Liu, Y. et al. Exploiting noncovalently conformational locking as a design strategy for high performance fused-ring electron acceptor used in polymer solar cells. J. Am. Chem. Soc. 139, 3356-3359 (2017).

3. Baran, D. et al. Reduced voltage losses yield $10 \%$ efficient fullerene free organic solar cells with $>1$ V open circuit voltages. Energy Environ. Sci. 9, 3783-3793 (2016).

4. Ma, X. et al. Efficient ternary polymer solar cells with two well-compatible donors and one ultranarrow bandgap nonfullerene acceptor. Adv. Energy Mater. 8, 1702854 (2018)

5. Zhan, L. et al. A near-infrared photoactive morphology modifier leads to significant current improvement and energy loss mitigation for ternary organic solar cells. Adv. Sci. 5, 1800755 (2018).

6. Nian, L. et al. Ternary non-fullerene polymer solar cells with $13.51 \%$ efficiency and a record-high fill factor of 78.13\%. Energy Environ. Sci. 11, 3392-3399 (2018).

7. Zhou, Z. et al. High-efficiency small-molecule ternary solar cells with a hierarchical morphology enabled by synergizing fullerene and non-fullerene acceptors. Nat. Energy 3, 952-959 (2018).

8. Zhang, M. et al. Efficient ternary non-fullerene polymer solar cells with PCE of $11.92 \%$ and FF of 76.5\%. Energy Environ. Sci. 11, 841-849 (2018).

9. Zheng, Z. et al. A highly efficient non-fullerene organic solar cell with a fill factor over 0.80 enabled by a fine-tuned hole-transporting layer. Adv. Mater. 30, 1801801 (2018).

10. Qian, D. et al. Design rules for minimizing voltage losses in high-efficiency organic solar cells. Nat. Mater. 17, 703-709 (2018)

11. Gao, K. et al. Deep absorbing porphyrin small molecule for high-performance organic solar cells with very low energy losses. J. Am. Chem. Soc. 137, $7282-7285$ (2015).

12. Zhu, L. et al. Aggregation-induced multilength scaled morphology enabling $11.76 \%$ efficiency in all-polymer solar cells using printing fabrication. $A d v$. Mater. 31, 1902899 (2019).

13. Liu, F. et al. Characterization of the morphology of solution-processed bulk heterojunction organic photovoltaics. Prog. Polym. Sci. 38, 1990-2052 (2013).

14. Chen, W., Nikiforov, M. P. \& Darling, S. B. Morphology characterization in organic and hybrid solar cells. Energy Environ. Sci. 5, 8045-8074 (2012).

15. Yuan, J. et al. Single-junction organic solar cell with over $15 \%$ efficiency using fused-ring acceptor with electron-deficient core. Joule 3, 1140-1151 (2019).

16. Fan, B. et al. Achieving over $16 \%$ efficiency for single-junction organic solar cells. Sci. China Chem. 62, 746-752 (2019).

17. Cui, Y. et al. Over $16 \%$ efficiency organic photovoltaic cells enabled by a chlorinated acceptor with increased open-circuit voltages. Nat. Commun. 10, 2515 (2019).
18. Yu, R. et al. Improved charge transport and reduced nonradiative energy loss enable over $16 \%$ efficiency in ternary polymer solar cells. Adv. Mater. 31, 1902302 (2019)

19. Zhu, L. et al. Efficient organic solar cell with $16.88 \%$ efficiency enabled by refined acceptor crystallization and morphology with improved charge transfer and transport properties. Adv. Energy Mater. 10, 1904234 (2020).

20. Fan, Q. et al. Chlorine substituted 2D-conjugated polymer for highperformance polymer solar cells with $13.1 \%$ efficiency via toluene processing. Nano Energy 48, 413-420 (2018).

21. Ma, L. et al. A ternary organic solar cell with $300 \mathrm{~nm}$ thick active layer shows over 14\% efficiency. Sci. China Chem. 63, 21-27 (2020).

22. Schlaf, R., Parkinson, B. A., Lee, P. A., Nebesny, K. W. \& Armstrong, N. R. $\mathrm{HOMO} / \mathrm{LUMO}$ alignment at PTCDA/ZnPc and PTCDA/CIInPc heterointerfaces determined by combined UPS and XPS measurements. J. Phys. Chem. B 103, 2984-2992 (1999).

23. Koster, L. J. A., Mihailetchi, V. D., Ramaker, R. \& Blom, P. W. M. Light intensity dependence of open-circuit voltage of polymer:fullerene solar cells. Appl. Phys. Lett. 86, 123509 (2005).

24. Xiao, B. et al. High efficiency organic solar cells based on amorphous electrondonating polymer and modified fullerene acceptor. Nano Energy 39, 478-488 (2017).

25. Schilinsky, P., Waldauf, C. \& Brabec, C. J. Recombination and loss analysis in polythiophene based bulk heterojunction photodetectors. Appl. Phys. Lett. 81, 3885-3887 (2002).

26. Carbone, A., Kotowska, B. K. \& Kotowski, D. Space-charge-limited current fluctuations in organic semiconductors. Phys. Rev. Lett. 95, 236601 (2005).

27. Blom, P. W. M., Tanase, C., de Leeuw, D. M. \& Coehoorn, R. Thickness scaling of the space-charge-limited current in poly(p-phenylene vinylene). Appl. Phys. Lett. 86, 092105 (2005).

28. Blom, P. W. M., Mihailetchi, V. D., Koster, L. J. A. \& Markov, D. E. Device physics of polymer:fullerene bulk heterojunction solar cells. Adv. Mater. 19, 1551-1566 (2007).

29. Fan, Q. et al. Synergistic effects of side-chain engineering and fluorination on small molecule acceptors to simultaneously broaden spectral response and minimize voltage loss for $13.8 \%$ efficiency organic solar cells. Sol. RRL 3, 1900169 (2019).

30. Weng, K. et al. Ternary organic solar cells based on two compatible PDI-based acceptors with an enhanced power conversion efficiency. J. Mater. Chem. A 7, 3552-3557 (2019).

31. Juška, G., Arlauskas, K., Stuchlik, J. \& Österbacka, R. Non-Langevin bimolecular recombination in low-mobility materials. J. Non-Cryst. Solids $\mathbf{3 5 2}$, $1167-1171$ (2006).

32. Kirchartz, T., Mattheis, J. \& Rau, U. Detailed balance theory of excitonic and bulk heterojunction solar cells. Phys. Rev. B 78, 235320 (2008).

33. Ihly, R. et al. Tuning the driving force for exciton dissociation in single-walled carbon nanotube heterojunctions. Nat. Chem. 8, 603-609 (2016).

34. Habisreutinger, S. N., Noel, N. K., Larson, B. W., Reid, O. G. \& Blackburn, J. L. Rapid charge-transfer cascade through SWCNT composites enabling lowvoltage losses for perovskite solar cells. ACS Energy Lett. 4, 1872-1879 (2019).

35. Larson, B. W. et al. Inter-fullerene electronic coupling controls the efficiency of photoinduced charge generation in organic bulk heterojunctions. $A d v$. Energy Mater. 6, 1601427 (2016).

36. Burlingame, Q. et al. Intrinsically stable organic solar cells under high intensity illumination. Nature 573, 394-397 (2019).

37. Bakulin, A. A. et al. The role of driving energy and delocalized states for charge separation in organic semiconductors. Science 335, 1340 (2012).

38. Ziffer, M. E. et al. Long-lived, non-geminate, radiative recombination of photogenerated charges in a polymer/small-molecule acceptor photovoltaic blend. J. Am. Chem. Soc. 140, 9996-10008 (2018).

39. Liu, Y., Zuo, L., Shi, X., Jen, A. K. Y. \& Ginger, D. S. Unexpectedly slow yet efficient picosecond to nanosecond photoinduced hole-transfer occurs in a polymer/nonfullerene acceptor organic photovoltaic blend. ACS Energy Lett. 3, 2396-2403 (2018).

40. Zhong, Y. et al. Molecular helices as electron acceptors in high-performance bulk heterojunction solar cells. Nat. Commun. 6, 8242 (2015).

41. Li, K. et al. Influence of exciton diffusion and charge-transfer state dissociation efficiency on the short-circuit current densities in semi-random donor/ acceptor polymer:fullerene solar cells. J. Phys. Chem. C 117, 6940-6948 (2013).

42. Kaake, L. G., Moses, D. \& Heeger, A. J. Coherence and uncertainty in nanostructured organic photovoltaics. J. Phys. Chem. Lett. 4, 2264-2268 (2013).

43. Vandewal, K., Tvingstedt, K., Gadisa, A., Inganäs, O. \& Manca, J. V. Relating the open-circuit voltage to interface molecular properties of donor:acceptor bulk heterojunction solar cells. Phys. Rev. B 81, 125204 (2010).

44. Tvingstedt, K. et al. Electroluminescence from charge transfer states in polymer solar cells. J. Am. Chem. Soc. 131, 11819-11824 (2009).

45. Yao, J. et al. Quantifying losses in open-circuit voltage in solution-processable solar cells. Phys. Rev. Appl. 4, 014020 (2015). 
46. Eisner, F. D. et al. Hybridization of local exciton and charge-transfer states reduces nonradiative voltage losses in organic solar cells. J. Am. Chem. Soc. 141, 6362-6374 (2019).

47. Marcus, R. A. Relation between charge transfer absorption and fluorescence spectra and the inverted region. J. Phys. Chem. 93, 3078-3086 (1989).

48. Benduhn, J. et al. Intrinsic non-radiative voltage losses in fullerene-based organic solar cells. Nat. Energy 2, 17053 (2017).

49. Song, J. et al. Morphology characterization of bulk heterojunction solar cells. Small Methods 2, 1700229 (2018)

50. Zhang, M., Zhu, L., Qiu, C., Zhang, Y. \& Liu, F. Printing and in situ morphology detection of organic thin film photovoltaic devices. Acta Polym. Sin. 50, 352-358 (2019).

51. Li, J. \& Ji, L. Adjusting multiple testing in multilocus analyses using the eigenvalues of a correlation matrix. Heredity 95, 221-227 (2005).

52. Saad, Z. S. et al. A new method for improving functional-to-structural MRI alignment using local Pearson correlation. Neuroimage 44, 839-848 (2009).

\section{Acknowledgements}

This work was financially supported by the grant from the National Natural Science Foundation of China (Grant Nos. 51973110, 21734009, 21905102, and 61805138), Beijing National Laboratory for Molecular Sciences (BNLMS201902), the Center of Hydrogen Science, Shanghai Jiao Tong University, China. We thank Cheng Wang and Chenhui Zhu from Advanced Light Source for providing $\mathrm{x}$-ray scattering tests, which were carried out at beamline 7.3.3 and 11.0.1.2 (a portion of this work) at the Advanced Light Source, Molecular Foundry, Lawrence Berkeley National Laboratory, supported by the DOE, Office of Science, and Office of Basic Energy Sciences. L.Z. acknowledge the funding supported by China Postdoctoral Science Foundation (Grant No. 2020M681278). Z.M. acknowledges the funding supported by the Natural Science Foundation of Shanghai (Grant No. 19ZR1401400). H.Z. acknowledges the funding supported by the National Key Research and Development Program of China (2017YFA0207700). T.P.R. and Q.H. were supported by the US Office of Naval Research under contract N00014-17-1-2244. A portion of this work was authored by the National Renewable Energy Laboratory, operated by Alliance for Sustainable Energy, LLC, for the U.S. Department of Energy (DOE) under Contract No. DE-AC36-08GO28308. B.W.L. acknowledges funding from the Solar Energy Technology Office (SETO), Office of Energy Efficiency and Renewable Energy, U.S. DOE, for microwave conductivity measurements. The views expressed in the article do not necessarily represent the views of the DOE or the U.S. Government.

\section{Author contributions}

F.L. and T.P.R. conceived and directed the study. M.Z. fabricated the BHJ devices and did morphology optimization. L.Z. characterized the BHJ devices and conducted the certification. TA results and corresponding analysis were provided by G.Z. and H.Z. Energy loss test was performed by T.H., and assisted by Z.M. and Z.T. C.Q. and Y.Z carried out recombination analysis. Z.Z. did correlation analysis. Q.H. assisted in scattering measurement and device performance certification. B.W.L. carried out TRMC test and W.F. provided TEM test. This manuscript was mainly prepared by F.L., M.Z., and L.Z., and all authors participated in the manuscript preparation and commented on the manuscript.

\section{Competing interests}

The authors declare no competing interests.

\section{Additional information}

Supplementary information is available for this paper at https://doi.org/10.1038/s41467020-20580-8.

Correspondence and requests for materials should be addressed to F.L.

Peer review information Nature Communications thanks the anonymous reviewer(s) for their contribution to the peer review of this work.

Reprints and permission information is available at http://www.nature.com/reprints

Publisher's note Springer Nature remains neutral with regard to jurisdictional claims in published maps and institutional affiliations.

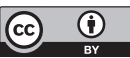

Open Access This article is licensed under a Creative Commons Attribution 4.0 International License, which permits use, sharing, adaptation, distribution and reproduction in any medium or format, as long as you give appropriate credit to the original author(s) and the source, provide a link to the Creative Commons license, and indicate if changes were made. The images or other third party material in this article are included in the article's Creative Commons license, unless indicated otherwise in a credit line to the material. If material is not included in the article's Creative Commons license and your intended use is not permitted by statutory regulation or exceeds the permitted use, you will need to obtain permission directly from the copyright holder. To view a copy of this license, visit http://creativecommons.org/ licenses/by/4.0/.

(C) The Author(s) 2021 